\title{
EVALUACIÓN DEL DAÑO POR CORROSIÓN DEL ACERO AISI SAE 4330 EN CONTACTO CON UN LODO DE PERFORACIÓN
}

Hugo Armando Estupiñán Duran ${ }^{1 *}$, Darío Yesid Peña Ballesteros², Vladimir Elles Macías ${ }^{3}$

*A quien debe dirigirse la correspondencia

\section{RESUMEN}

Los lodos de perforación son de suma importancia en la industria del petróleo, estos se pueden encontrar en forma base aceite o base agua, los base aceite no son los más adecuados por los problemas ambientales que genera y los base agua presentan alta corrosividad. Estos lodos tienen como función llevar a superficie los recortes de perforación, el enfriamiento, lubricación de la broca y de las sartas de perforación, como también mantener la estabilidad del pozo y evitar algún escape de gas o crudo. Es de esperarse que los lodos no ocasionen un excesivo desgaste ni corrosión en los materiales que componen los equipos de perforación. En este trabajo se ha evaluado la corrosión de un acero AISI 4330 en contacto con un lodo de perforación base agua, por medio de técnicas electroquímicas de extrapolación de Tafel, Espectroscopia de impedancia, Resistencia a la polarización. Los ensayos de corrosión fueron realizados a temperaturas de 30,45 y $60^{\circ} \mathrm{C}$ y velocidades de rotación de 1000 , 2000, $3000 \mathrm{rpm}$ en electrodo de cilindro rotatorio ECR, simulando condiciones de operación en fondo de pozo de perforación y de equipo de superficie de tratamiento de lodos. Se encontró que la variable más influyente en el proceso de corrosión, fue la velocidad de rotación, que influyó en un aumento de la densidad de corriente de corrosión, incrementando la velocidad de corrosión. De igual forma, se corroboró este comportamiento con la disminución de la resistencia de la polarización al aumentar la velocidad de rotación.

Palabras clave: Acero AISI 4330, lodo de perforación, resistencia a la polarización, técnicas electroquímicas., velocidad de corrosión,

\section{CORROSION ASSESSMENT OF AISI SAE 4330 STEEL IN CONTACT WITH DRILLING MUD}

\begin{abstract}
Drilling fluids also known as drilling muds play a very important role in the oil and gas industry. Muds can be oil-based or water-based. Oil-based fluids have limited application due environmental problems. On the other hand, water-based muds are usually corrosive. Drilling muds have different functions such as the transport of drill cuttings to the surface, cooling, cleaning and lubrication of the drill bit and the drill collars as well as maintaining the stability of the well and the prevention of any escape of gas or oil. It is expected that the mud will not cause excessive wear or corrosion to the materials used in the drillstring. In this study the corrosion of the AISI 4330 steel in contact with a water-based drilling mud was evaluated using the electrochemical techniques Tafel extrapolation and impedance spectroscopy. The corrosion tests were performed at temperatures of 30,45 and $60^{\circ} \mathrm{C}$ and rotation rates of 1000, 2000, $3000 \mathrm{rpm}$ using a rotating cylinder electrode (ECR). Therefore, downhole and surface service conditions were simulated. It was found that the rotation rate is the most influential variable on the corrosion rate of the AISI 4330 steel. With increasing the rotation rate the corrosion current density increased and the polarization resistance decreased, consequently, the corrosion rate of the investigated steel increased
\end{abstract}

Keywords: AISI SAE 4330 steel, corrosion rate, drilling mud, electrochemical techniques, polarization resistance.

1. PhD, Universidad Nacional de Colombia, calle $75 \mathrm{~N}^{\circ} 79^{\mathrm{a}}-51$ Facultad de Minas, sede Medellín, Colombia.

2. $\mathrm{PhD}$, Universidad Industrial de Santander, carrera 27 calle 9 ciudad universitaria, Bucaramanga, Colombia.

3. Ing., Universidad Industrial de Santander, carrera 27 calle 9 ciudad universitaria, Bucaramanga, Colombia. 


\section{INTRODUCCIÓN}

En las operaciones de perforación se utilizan lodos, que dentro de sus finalidades está la de por densidad suspender los recortes y llevarlos a superficie, el enfriamiento, la limpieza y la lubricación de la broca y de las sartas de perforación, como también mantener la estabilidad del agujero y evitar algún escape de gas o crudo. Cabe resaltar que el éxito de la perforación depende del rendimiento del lodo que se hace circular. Dentro de las propiedades que se esperan de los lodos están la de no causar desgaste excesivo en los equipos o corroerlos. Uno de los problemas que presentan los lodos es la variación de su composición a medida que se avanza en la perforación, por lo cual no se puede determinar un rendimiento continúo en la perforación.

Los lodos de perforación base aceite tienen un buen comportamiento en operaciones de perforación, por cuanto son menos corrosivos, sin embargo debido a su costo, a la contaminación que produce y a las reglamentaciones ambientales, se han formado menos atractivos para la industria de perforación. Los lodos de perforación base agua son los más baratos, disponibles y sus propiedades pueden ser más fácilmente mantenidas y controladas, como por ejemplo en la variación de $\mathrm{pH}$. El rango de $\mathrm{pH}$ de los lodos de perforación varia generalmente entre 7,5 y 10,5 lo cual ayuda a mantener los materiales utilizados en operación en un estado pasivo o de no corrosión, valores por debajo o por encima de este rango aumentan la tendencia de estos materiales a la corrosión, un problema que se genera mediante la utilización de lodos base agua es su alta corrosividad, debido a que estos presentan una alta fracción de agua que entra en contacto con la sarta de perforación y los equipos de superficie. Cuando la sarta de perforación entra en contacto con el lodo, los gases disueltos en éste (oxígeno, dióxido de carbono), producen problemas graves de corrosión llevando a la falla del material y pérdidas económicas. Los efectos de la corrosión sobre instalaciones y equipos industriales produce anualmente pérdidas que llegan a cifras muy importantes: en los países industrializados se han valorado en un 3\% del PBI. Este porcentaje puede tomarse sobre la valoración equivalente de la industria petrolera y del gas, para llegar a una cuantificación aproximada de sus efectos económicos. De todas las fallas que ocurren en las operaciones de la industria del gas y del petróleo la más importante es la corrosión en un 33\% de los casos, tal y como puede constatarse en un trabajo realizado por Kermany y Harrop de la BP (Rey, 2008) ${ }^{[5]}$.

Se ha estudiado el efecto del desprendimiento de las capas de óxido de cromo por fuerzas de fricción generadas en la operación de perforación, en un rotor en contacto con lodo, considerando diferentes concentraciones de $\mathrm{NaCl}$ encontrándose que la solución salina penetra por los poros formados en la superficie de las capas, producto de la fricción (Cobo, Suárez Baldo, \& Bessone, 1999).

Tomoe y colaboradores en 1999, estudiaron el mecanismo de corrosión por los efectos combinados de oxígeno disuelto y dióxido de carbono presente por la degradación térmica de los lodos sintéticos para temperaturas superiores a $180^{\circ} \mathrm{C}$, encontrando daños de corrosión generados por una celda de aireación diferencial causada por la acumulación de minerales de arcilla en los sitios anódicos (Tomoe, Shimizu, \& Nagae, n.d.). En 2003, Anguiño y Keitelman, reportaron el análisis de falla en columna de perforación donde evaluaron la velocidad de corrosión en un acero con diferentes $\mathrm{pH}$, con condiciones aireadas, desaireadas y diferentes atmosferas de presión, determinando variaciones de velocidad de corrosión y daño encontrado por la presencia de $\mathrm{HCl}$ en los lodos. En 2011, Sarhan y Kadhim, estudiaron la corrosión de un acero de carbono en contacto de lodos base agua a diferentes temperaturas y concentraciones de sales, encontrando que la corrosión disminuyó con el aumento de la concentración de sal e incremento de la corrosión con la temperatura (Kadhim, 2011)the most widely used engineering material, accounts for approximately $85 \%$, of the annual steel production worldwide. Despite its relatively limited corrosion resistance, carbon steel is used in large tonnages in marine applications, nuclear power and fossil fuel power plants, transportation, chemical processing, petroleum production and refining, pipelines, mining, construction and metal-processing equipment. This paper Investigate Carbon steel corrosion in water. The corrosion rate in production and casing pipes in water base drilling mud (packer $<$ span dir $=|\gg r t||\gg\rangle<<$ span $>$ fluid. Farzam y colaboradores, estudiaron mediante pruebas potenciodinámicas la velocidad de corrosión en aceros API A5, API 5L y AISI 1020, realizando variaciones en la composición de los lodos, encontrando comportamientos de picado en los aceros debido a la disminución del pH en los lodos (Farzam, Baghery, \& Dezfully, 2011). 
En este trabajo se determinó la velocidad de corrosión en un acero AISI 4340 expuesto a lodos de perforación base agua, a diferentes velocidades y temperaturas de trabajo, mediante técnicas electroquímicas Tafel, espectroscopia de impedancia electroquímica (EIE) y resistencia a la polarización lineal (RPL) en Electrodo de Cilindro Rotatorio (ECR).

\section{MATERIALES Y MÉTODOS}

El acero AISI SAE 4330 usado para este trabajo, fue expuesto a lodos de perforación tomados a profundidades de 1000,2000 y 3000 pies y a temperaturas de 30,45 y $60^{\circ} \mathrm{C}$. Se utilizaron lodos del campo de producción la Cira Infantas, suministrado por una empresa de Perforación, y el acero fue suministrado por la empresa Materials Xperts Ltda. Este lodo, preparado con arcilla, bentonita, sulfato de bario, cloruro de sodio, con lecturas de contenido de cloruro de hasta 15.000 p.p.m., fue retirado a diferentes profundidades de perforación.

Se fabricaron probetas con el acero AISI SAE 4330, a partir de un Shock Absorber, las características de esta sarta son las de su alto peso, dureza, capacidad de soportar el impacto en la perforación ya que sostiene la broca de perforación. En la Figura 1 se muestran las probetas montadas en el eje del cilindro rotatorio. La preparación de las probetas se llevó a cabo mediante la aplicación de la norma ASTM G1-90. Las probetas de acero AISI-SAE 4330 se entregaron desde su maquinado, puliendo con papel de carburo de silicio número 600 , seguido por un desengrasado en etanol y un posterior secado con chorro de aire caliente seco.

Las dimensiones de la probeta fueron de $12 \mathrm{~mm}$ diámetro externo, $6 \mathrm{~mm}$ de diámetro interno y $8 \mathrm{~mm}$ de altura, con un área superficial de trabajo de $3 \mathrm{~cm}^{2}$.

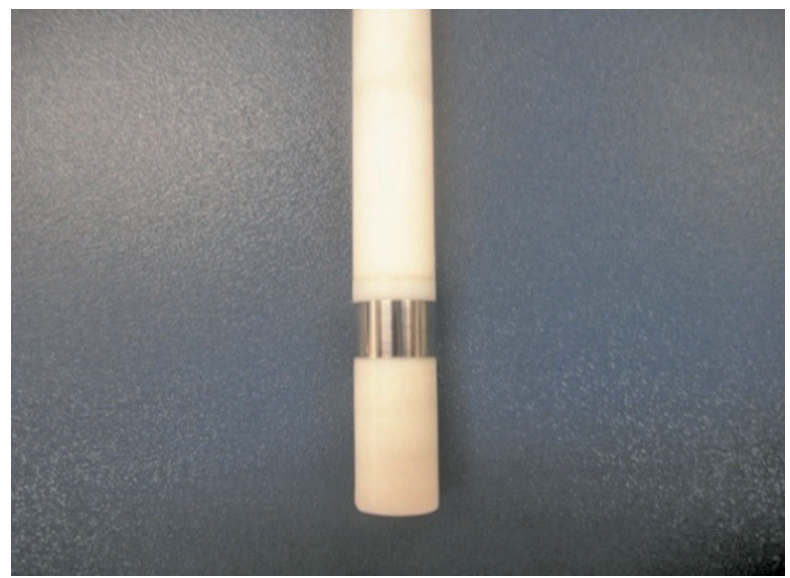

Figura 1. Eje del cilindro rotatorio con la probeta preparada
La composición del acero la suministró la empresa Materials Xperts Ltda., la cual se puede observar en la Tabla 1.

Tabla 1.Composición porcentual del acero AISI SAE 4330

\begin{tabular}{|cc|}
\hline $\mathrm{C}$ & 0.31 \\
$\mathrm{Mn}$ & 0.88 \\
$\mathrm{P}$ & 0.011 \\
$\mathrm{~S}$ & 0.003 \\
\hline $\mathrm{Si}$ & 0.31 \\
\hline $\mathrm{Ni}$ & 1.74 \\
$\mathrm{Cr}$ & 0.88 \\
\hline $\mathrm{Mo}$ & 0.41 \\
\hline $\mathrm{Cu}$ & 0.26 \\
\hline $\mathrm{Sn}$ & 0.011 \\
\hline $\mathrm{Al}$ & 0.034 \\
$\mathrm{~V}$ & 0.061 \\
\hline
\end{tabular}

La superficie de la probeta, mostrada en la Figura 2, se pulió según la norma ASTM E03, hasta 0.05 micras, realizando ataque con Nital al 2\%, se observó una microestructura de martensita revenida debido al tratamiento térmico de temple y revenido que la precede, además de unos pequeños precipitados de sulfuro de manganeso, los cuales están en los niveles permisibles por la norma ASTM E45.

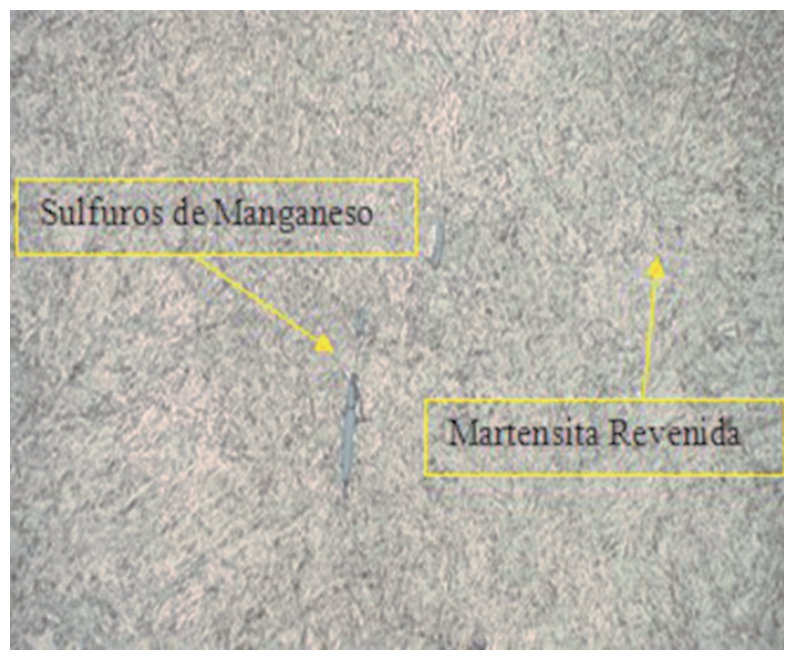

Figura2. Microestructura del Acero AISI SAE 4330, A $1400 \mathrm{X}$

Las pruebas electroquímicas se realizaron mediante un potenciostato/Galvanostato ACM GILL, usado para la medición de las pruebas electroquímicas. El montaje se observa en la Figura 3. 


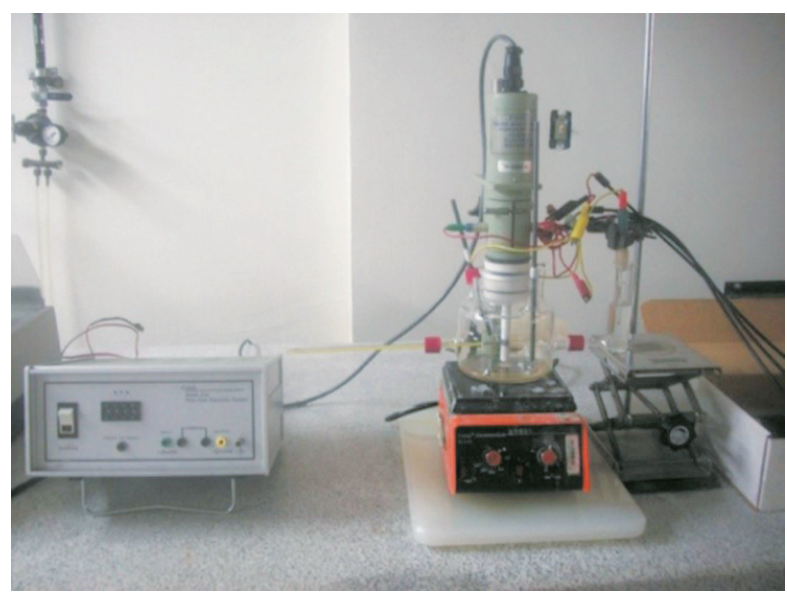

Figura 3. Montaje para la realización de las pruebas ECR

Para la realización de los experimentos, se tomó las variables que se podían controlar en este sistema, como fue la velocidad de rotación del electrodo y la temperatura respectiva de cada lodo tomado a las diferentes profundidades. En este artículo se muestran los resultados obtenidos de las variaciones de velocidad de rotación correspondientes a la máxima profundidad tomada, simulando condiciones hidrodinámicas respectivas.

Los ensayos se realizaron en este orden: primero, se dejó estabilizar el potencial de corrosión, y luego se corrió la prueba de Resistencia a la Polarización, seguido de Espectroscopia de Impedancia Electroquímica y finalmente la prueba de Extrapolación Tafel. Posteriormente se realizó análisis de las superficies de las probetas mediante SEM después de la realización de los ensayos y con los resultados obtenidos de Resistencia a la Polarización se realizó un análisis estadístico para determinar la variable más influyen en el proceso.

\section{ANÁLISIS Y DISCUSIÓN DE RESULTADOS}

En la Figura 4 se observa la variación de rotación del electrodo de cilindro rotatorio a una temperatura de $45^{\circ} \mathrm{C}$

Las gráficas de LPR muestran un decremento en la resistencia a la polarización al aumentar la velocidad de rotación de $1000 \mathrm{rpm}$ a $3000 \mathrm{rpm}$; sin embargo se muestra un incremento de la estabilidad, de acuerdo a los valores de potencial, siendo más altos a velocidades rotacionales más altas. Los factores que pueden afectar este comportamiento han sido determinados por varios investigadores: a) Incremento en la velocidad de reacción química b) Disminución de la solubilidad de los gases en el agua, factor que se refiere a la hidrodinámica del proceso, que será tenido en cuenta en estudios posteriores c) Disminución de la viscosidad de la solución, d) Incremento en el coeficiente de difusión de las especies reaccionantes. Con el incremento de la temperatura, se observó una disminución en la resistencia a la polarización a las diferentes velocidades de rotación. Resultados similares con un fluido salmueraaceite y con un acero AISI-SAE 1020 fueron obtenidos por otros investigadores anteriormente. (Melgarejo \& Muñoz, 2007)

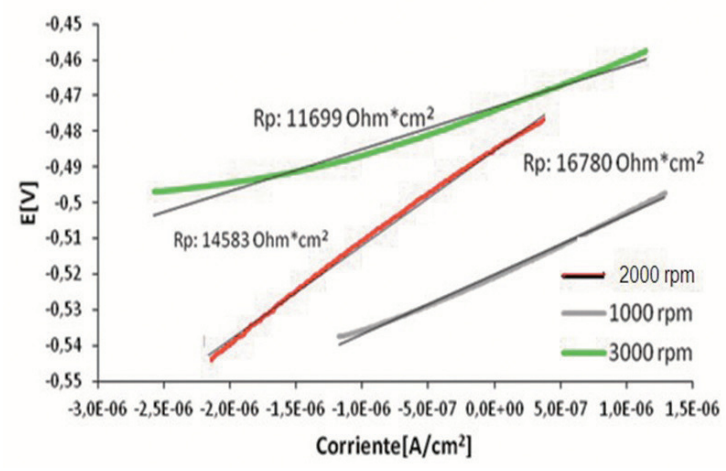

Figura 4. Curva de LPR para un Acero AISI 4330, en lodo de perforación a velocidades de rotación de 1000 , 2000 y $3000 \mathrm{rpm}$ a una temperatura de $45^{\circ} \mathrm{C}$.

En la Figura 5 se muestra la variación de la temperatura en ensayos realizados de Tafel a 30 y $60^{\circ} \mathrm{C}$ a una velocidad de rotación de $1000 \mathrm{rpm}$. Se observa una incremento en la densidad de corriente de corrosión al aumentar la temperatura y de acuerdo a la morfología de las curvas, es importante resaltar que de $30^{\circ} \mathrm{C}$ a $60^{\circ} \mathrm{C}$ se presentó diferencias en el mecanismo de corrosión, pasando de un control por transferencia de carga a un control por transferencia de masa lo cual se hace evidente por una mayor verticalidad de la curva catódica de la prueba realizada a $60^{\circ} \mathrm{C}$. Un aumento en la densidad de corriente de corrosión fue observado al aumentar la velocidad de rotación del electrodo de cilindro rotatorio, a cada temperatura de ensayo. 


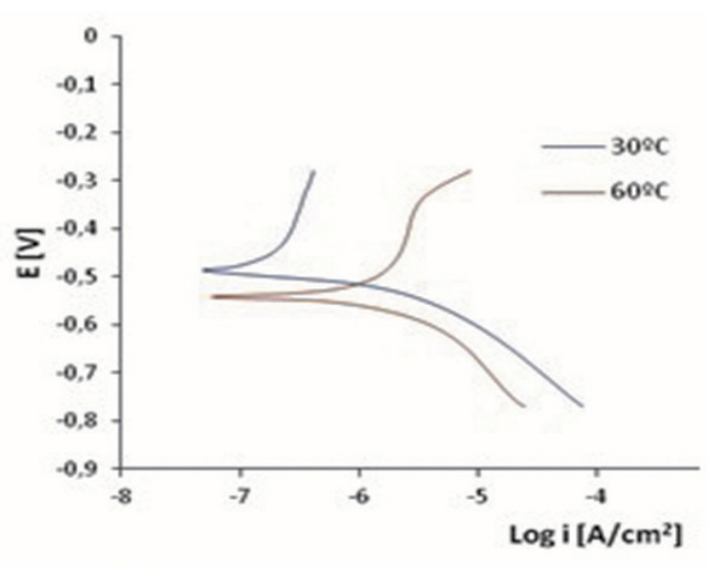

Figura 5. Curvas Tafel para un Acero AISI 4330 en lodo de perforación a una velocidad de rotación de $1000 \mathrm{rpm}$ a 30 y $60^{\circ} \mathrm{C}$.

En la Figura 6 se muestra la variación de la temperatura en ensayos realizados de espectroscopia de impedancia electroquímica a 30 y $60^{\circ} \mathrm{C}$ a una velocidad de rotación de $1000 \mathrm{rpm}$.

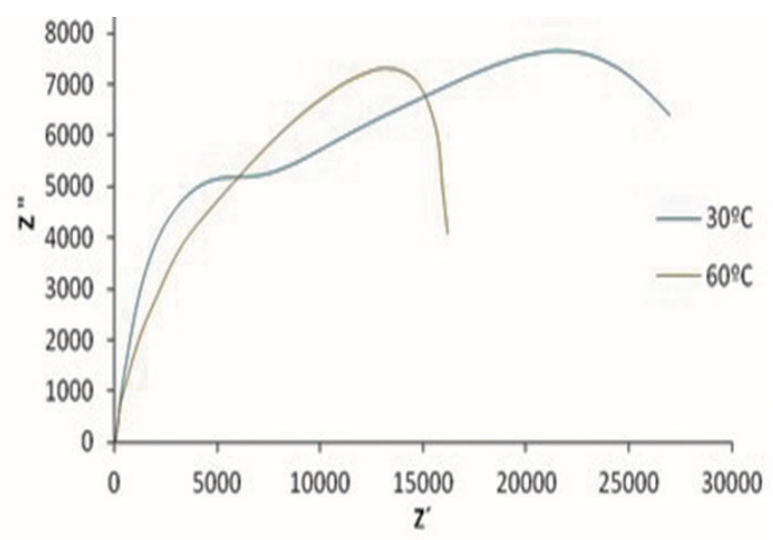

Figura 6. Curvas de Nyquist para un Acero AISI 4330, en lodo de perforación a una velocidad de rotación de $1000 \mathrm{rpm}$ a 30 y $60^{\circ} \mathrm{C}$.
Se observa dos relajaciones a la transferencia de carga, separadas aproximadamente a $1000 \mathrm{~Hz}$, con un incremento en la transferencia de masa al incrementar la temperatura y una disminución de la resistencia a la polarización respectiva, corroborando lo observado en las curvas de RPL y Tafel. Estos comportamientos son resistivos y capacitivos para $30^{\circ} \mathrm{C}$ y $60^{\circ} \mathrm{C}$ respectivamente lo cual es evidentemente comprobado por la depresión en los semiarcos en el espectro de Nyquist. Este comportamiento es característico de afectación por corrosión uniforme, donde los productos de corrosión son afectados por la temperatura, la cual incrementa la movilidad iónica y la inestabilidad de los óxidos formados sobre la superficie del acero.

Un comportamiento similar al observado en la Figura 6 se presentó al variar la velocidad de rotación del electrodo de cilindro rotatorio a cada temperatura de trabajo.

La presencia de dos semicírculos en el ensayo a $30{ }^{\circ} \mathrm{C}$ es característico de proceso de corrosión por control mixto, de transferencia de masa (semiarco a bajas frecuencias) y transferencia de carga (semiarco a altas frecuencias)

En la Tabla 2 se observan los resultados de densidad de corriente de corrosión y de velocidad de corrosión a las condiciones de trabajo expuestas.

Donde:

$$
B=\frac{\beta_{a} \beta_{c}}{2,3 * I_{\text {corr }}\left(\beta_{a}+\beta_{c}\right)}
$$

Siendo $\beta a$ la pendiente anódica; $\beta c$ la pendiente catódica; $I_{\text {corr }}$ la corriente de corrosión y B el coeficiente de SternGeary de acuerdo a la norma ASTM-G-102.

Tabla 2. Datos de corrosión obtenidos mediante la técnica de extrapolación Tafel

\begin{tabular}{|ccccccc} 
Prueba & & $\begin{array}{c}\mathrm{I}_{\text {corr }} \\
{\left[\mu \mathrm{A} / \mathrm{cm}^{2}\right]}\end{array}$ & $\begin{array}{c}\mathrm{B}_{\mathrm{c}} \\
{[\mathrm{V} / \mathrm{dec}]}\end{array}$ & $\begin{array}{c}\mathrm{B}_{\mathrm{a}} \\
{[\mathrm{V} / \mathrm{dec}]}\end{array}$ & $\mathrm{B}$ & Vel Corr/ Mpy \\
\hline $100030^{\circ} \mathrm{C}$ & $\mathrm{rpm}$ & $4,46 \times 10^{-2}$ & $6,63 \times 10^{-2}$ & $8,23 \times 10^{-2}$ & $1,02 \times 10^{-2}$ & $2,07 \times 10^{-1}$ \\
\hline $100060^{\circ} \mathrm{C}$ & $\mathrm{rpm}$ & $1,00 \times 10^{-1}$ & $9,87 \times 10^{-2}$ & $6,28 \times 10^{-2}$ & $1,66 \times 10^{-2}$ & $4,64 \times 10^{-1}$ \\
$300030^{\circ} \mathrm{C}$ & $\mathrm{rpm}$ & $8,96 \times 10^{-2}$ & $6,97 \times 10^{-2}$ & $4,72 \times 10^{-2}$ & $8,49 \times 10^{-3}$ & $4,15 \times 10^{-1}$ \\
\hline $300060^{\circ} \mathrm{C}$ & $\mathrm{rpm}$ & $9,96 \times 10^{-2}$ & $7,87 \times 10^{-2}$ & $4,34 \times 10^{-2}$ & $1,37 \times 10^{-2}$ & $4,62 \times 10^{-1}$ \\
$200045^{\circ} \mathrm{C}$ & $\mathrm{rpm}$ & $8,81 \times 10^{-2}$ & $9,81 \times 10^{-2}$ & $3,43 \times 10^{-2}$ & $1,10 \times 10^{-2}$ & $4,08 \times 10^{-1}$ \\
\hline
\end{tabular}


A partir de estos datos, se observa una mayor incidencia en la velocidad de corrosión con la variación de la velocidad de rotación. La existencia de iones $\mathrm{Cl}-$, que penetran y deterioran la posible capa protectora del óxido formado $\left(\mathrm{Fe}_{3} \mathrm{O}_{4}\right)$. Este óxido es protector. Sin embargo, la ausencia de este óxido en la superficie del electrodo de trabajo después de las pruebas, se debe a la hidrodinámica del fluido y la presencia de óxido de aluminio, óxido de silicio proveniente de los lodos de perforación, lo cual genera un desprendimiento de este óxido protector hacia el seno de la solución debido a la poca adherencia que presentó a altas velocidades de flujo (Melgarejo \& Muñoz, 2007).

En el análisis realizado mediante EDX para la prueba a $3000 \mathrm{rpm}-60^{\circ} \mathrm{C}$, Figura 7, se observó corrosión localizada, encontrándose óxidos formados. Con las relaciones de porcentaje de peso atómico dadas por los resultados de SEM-EDX, se puede establecer una relación estequiométrica aproximada con los porcentajes de oxígeno, hierro, silicio, aluminio y calcio, los cuales contribuyen en la corrosión o protección de la probeta.

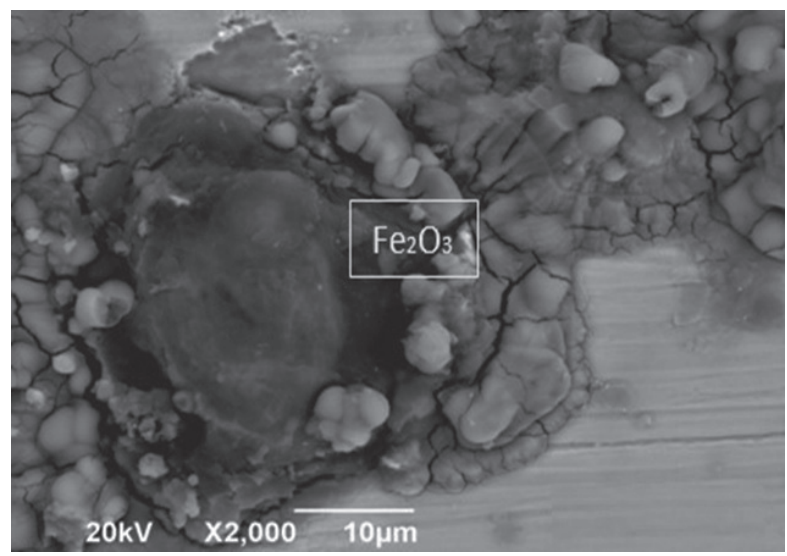

Figura 7. Presencia de precipitado de $\mathrm{Fe}_{2} \mathrm{O}_{3}$. A $3000 \mathrm{rpm}$ $-60^{\circ} \mathrm{c}$

En la Figura 8, se muestra el análisis SEM-EDX en donde se encontró la posible presencia de óxido de silicio y óxido de aluminio los cuales son compuestos que tienen la mayor probabilidad de aparecer en esta microestructura según los señalado en la Figura 8, el cual influye en la abrasión y el desprendimiento de la capa protectora. El origen de este óxido se puede dar, en las formaciones rocosas donde se realizan las perforaciones con estos materiales. Debido a las velocidades bajas de corrosión, las cuales fueron calculadas para todos los ensayos y de acuerdo al análisis superficial, se puede decir que no hubo una formación continua de óxidos sobre el acero y que además, el efecto erosivo del lodo, impiden que esta capa, se presentara de una forma homogénea. En este orden de ideas, se hace necesario realizar posteriores estudios para cuantificar el daño por erosión.

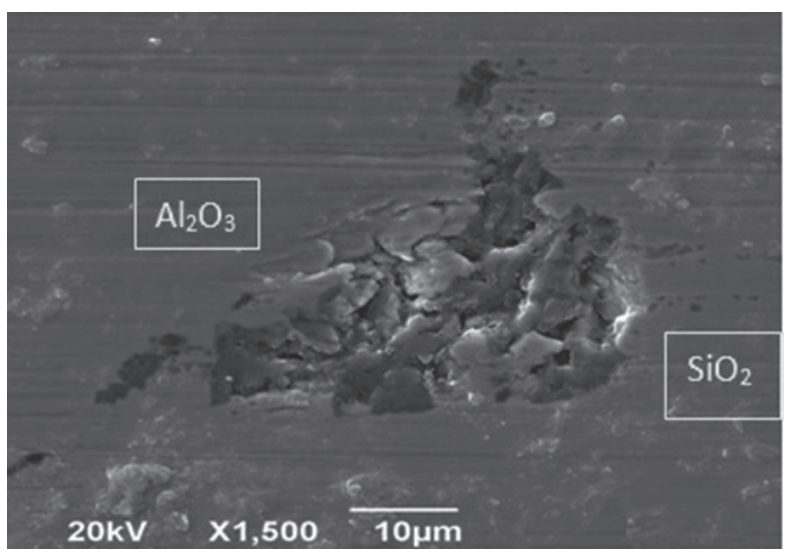

Figura 8. Presencia de $\mathrm{SiO}_{2}$ y $\mathrm{Al}_{2} \mathrm{O}_{3}$. A $3000 \mathrm{rpm}-60^{\circ} \mathrm{c}$

A altas velocidades de rotación, se encontró la presencia de daño localizado por picado y la falta de óxido protector sobre la superficie del acero. Este mismo comportamiento fue encontrado en un trabajo bajo condiciones similares (Wiese, Stevens, \& Sarmiento, 2013). Cabe anotar que para que se inicie el picado electroquímicamente, se debe alcanzar el potencial de picadura; ya que la composición de los lodos de perforación es fija pero cambiante en el contenido de cloruros, de acuerdo a la profundidad de la perforación según la dosificación del fabricante del lodo. Además, la heterogeneidad de material, dada en inclusiones, bandas, y cavidades subsuperficiales y superficiales, favorece la iniciación del picado. Por otro lado la deformación plástica en el acero dada por la erosión, también podría tener un efecto sobre la corrosión.

Cabe mencionar que el picado en aceros de baja aleación como el investigado, tiene lugar en lodos de perforación, cuando la capa de productos de corrosión (no precisamente protectora) es dañada localmente, por ejemplo, por efecto de los cloruros del fluido, a través de los esfuerzos de corte o por partículas en el fluido, dando lugar a zonas preferenciales para el picado. La formación y daño de la capa de productos de corrosión estará por tanto estrechamente ligada al $\mathrm{pH}$ del lodo, a la temperatura, al contenido de cloruros y a la velocidad de flujo. 


\section{CONCLUSIONES}

Se determinó para un acero AISI 4330 expuesto a desgaste por el contacto con un lodo de perforación base agua y de acuerdo al análisis de los ensayos electroquímicos de corrosión, que entre las variables de velocidad de rotación y la temperatura del pozo, la que más influye en el proceso de degradación del acero, es la velocidad de rotación.

Debido a los valores bajos de corrosión hallados, se concluye que el lodo base agua a condiciones de trabajo con un $\mathrm{pH}$ de 10.3, son de excelentes características de desempeño para la exploración y explotación en la industria del petróleo, ya que no es corrosivos, respecto al acero AISI 4330 que normalmente es usado en este tipo de operación.

\section{REFERENCIAS}

1. Cobo, E. O., Suárez Baldo, R. A., \& Bessone, J. B. (1999). Corrosion of chromium plated rotor in drilling fluid. Surface and Coatings Technology, 122(1), 39-43. http://doi.org/10.1016/S02578972(99)00408-9

2. Farzam, M., Baghery, P., \& Dezfully, M. (2011). Corrosion Study of Steel API 5A, 5L and AISI 1080, 1020 in Drill-Mud Environment of Iranian
Hydrocarbon Fields. ISRN Materials Science, 2011. Retrieved from citeulike-article-id:9727816

3. Kadhim, F. S. (2011). Investigation of carbon steel corrosion in water base drilling mud. Modern Applied Science, 5(1), 224-229. http://doi.org/10.5539/mas. v5n $1 \mathrm{p} 224$

4. Melgarejo, C., \& Muñoz, L. (2007). Estudio de la velocidad de corrosión de un acero AISI-SAE 1020 en un sistema salmuera-aceite refinado-co2-h2s por medio de un electrodo de cilindro rotatorio (ECR) y un simulador de flujo., 55.

5. Rey, S. (2008). Evaluacion de la corrosión de una acero AISI SAE en un flujo multifasico salmueraaceite mineral $\mathrm{CO} 2-\mathrm{H} 2 \mathrm{~S}$, por medio de una simulador de flujo, 10-12.

6. Tomoe, Y., Shimizu, M., \& Nagae, Y. (n.d.). Unusual Corrosion of a Drill Pipe in Newly Developed Drilling Mud During Deep Drilling.

7. Wiese, G., Stevens, J., \& Sarmiento, H. (2013). Pitting Corrosion Resistance of CrMn Austenitic Stainless Steel in Simulated Drilling ConditionsRole of $\mathrm{pH}$, Temperature, and Chloride Concentration. Corrosion, 69(11), 1095-1102. http://doi.org/10.5006/0947

Recepción: 31 de Julio de 2015

Aceptación: 6 de Septiembre de 2015 